\title{
PARTICIPACIÓN CIUDADANA EN LA GESTIÓN PÚBLICA LECCIONES Y DESAFÍOS
}

\author{
Rafael Enrique Valenzuela Mendoza \\ Universidad Autónoma de Ciudad Juárez, México \\ rafael.valenzuela1@gmail.com
}

\section{RESUMEN}

El artículo sistematiza la discusión sobre participación ciudadana en la gestión pública desarrollada en los dos números especiales publicados por Revista de Gestión Pública durante 2019. Para esto, en primer lugar, se discute, desde una perspectiva teórica, la relación entre democracia, rendición de cuentas y gobernanza. Con posterioridad, el artículo evalúa las principales lecciones y desafíos identificados en los artículos publicados en los dos números especiales. En específico, se abordan las siguientes materias: 1) las modalidades de participación ciudadana; 2) la innovación democrática; y 3) el combate a la corrupción. Finalmente, se presenta una propuesta de agenda para el diseño de un sistema ciudadanizado de rendición de cuentas.

Palabras clave: Participación, Democracia, Rendición de cuentas, Innovación, Corrupción. 


\title{
CITIZEN PARTICIPATION IN PUBLIC. MANAGEMENT LESSONS AND CHALLENGES
}

\begin{abstract}
This article systematizes the discussion about citizen participation in public management that took place through the two special issues of Revista de Gestión Pública published in 2019. It first looks at the relationship between democracy, accountability and governance from a theoretical standpoint. It then evaluates the main lessons and challenges identified in the articles published in these two special issues. Specifically, it addresses the following topics: 1) modes of citizen participation; 2) democratic innovation; and 3) the combat of corruption. Finally, it proposes an agenda for the design of a citizen system of accountability.
\end{abstract}

Keywords: Participation, Democracy, Accountability, Innovation, Corruption. 


\section{INTRODUCCIÓN}

Durante el 2019, la Revista de Gestión Pública editó dos números especiales que abordaron, desde diversas miradas, la importancia de la participación ciudadana en la gestión pública. En las siguientes páginas, se discuten los principales hallazgos que estos trabajos entregan y se delinean desafíos futuros, tanto para las administraciones públicas latinoamericanas como para la comunidad académica.

¿Por qué es relevante la participación ciudadana en un sistema de rendición de cuentas? Para perfilar una respuesta a esta pregunta, es necesario explorar el terreno de la democracia y la consolidación de sus mecanismos e instrumentos que activan la ciudadanización. Para que la democracia funcione como un sistema de rendición de cuentas, es fundamental activar diversos mecanismos que faciliten distribuir el poder, crear controles y equilibrios entre los órganos que lo ejercen y lograr consolidar la vía electoral de acceso al poder. Para brindar legitimidad a los gobiernos electos, además, se requiere construir confianza en las instituciones, con base en una relación de mutuo entendimiento para combatir los problemas más importantes de la sociedad.

Una democracia como sistema de rendición de cuentas, se funda en ciudadanos que hablan con la verdad al poder político, pero la ecuación está incompleta si se dejan de promover los valores de la ética y los saberes cívicos y, además, si se desconoce la importancia de la vigilancia y el control externo y social sobre los gobiernos.

El objetivo del artículo es reflexionar la participación ciudadana en torno de tres grandes ejes de discusión: las modalidades de participación ciudadana, la innovación democrática y el combate a la corrupción. El marco de reflexión es un contexto de democracia con fuertes dificultades para regenerar confianza institucional, lo que sugiere que las acciones del Estado y de la ciudadanía deben enfocarse en construir bases para un sistema más relacional entre actores y grupos a favor de la rendición de cuentas.

\section{DEMOCRACIA, RENDICIÓN DE CUENTAS Y GOBERNANZA}

Tradicionalmente la democracia ha sustentado su defensa de dos principios fundamentales: libertad e igualdad. En ambos se presenta el dilema, ¿es posible la igualdad sin libertad? O, ¿existe la libertad sin igualdad? (Sartori 2003: 223-271). Estas preguntas conducen a una discusión que genera 
confrontación sobre posiciones ideológicas, pero también respecto a modelos de participación ciudadana.

El principio de libertad pone énfasis en limitar el poder Estado mediante el ejercicio de los derechos ciudadanos, mientras que la igualdad hace énfasis en la intervención del Estado para reducir brechas que genera la libre competencia. En este debate es fundamental adicionar un tercer principio a nuestra democracia, el control. El principio del control refiere a "el control por el gobierno, así como el control del gobierno" (Campbell 2008: 6). En un Estado de derecho, el control del gobierno implicará gobernabilidad, pero una intensa participación ciudadana favorecerá una gobernabilidad democrática, hasta llegar a un arreglo institucional donde los ciudadanos participan de la arena de la decisión pública. Por otra parte, el control del gobierno viene dado por la sociedad civil vigilante y que se involucra en el monitoreo de lo público y en las responsabilidades del gobierno para elevar la calidad del resultado de la gestión pública.

Lo anterior sugiere que un sistema de rendición de cuentas es la vía por la cual es posible fraguar los principios de libertad, igualdad y control, principios que están vinculados, necesariamente, a la rendición de cuentas (Morlino 2005). Los tres tipos de rendición de cuentas que una democracia necesita gestionar son los siguientes: 1) la electoral; 2) la societal; 3) la horizontal.

La rendición de cuentas electoral dispone de un instrumento fundamental como es el voto libre y secreto. Con este se elige a los representantes de los ciudadanos para el proceso de toma de decisiones en una democracia representativa. Cuando se trata de una democracia liberal, se presentan candidatos postulados por partidos políticos o candidatos sin partido, con el propósito de que los votantes valoren a sus gobiernos o las promesas de candidatos a puestos de elección. En una democracia menos liberal y más republicana, la participación ciudadana no se limita a una elección, sino que el voto es un instrumento de decisión a través de un referéndum o plebiscito.

Existe, en ambos casos, un conjunto de reglas electorales para la agregación de preferencias, donde la opción más votada es la que se llevará adelante. La rendición de cuentas electoral existe por definición en un régimen democrático y refiere a la posibilidad de sancionar con el voto ciudadano a los gobiernos que buscan la reelección o al candidato postulado por el partido en el poder (O’Donnell 2003). 
En tanto, la rendición de cuentas societal tiene una característica central: la lógica del control del poder desde la sociedad civil. Esto implica una forma relacional del ciudadano con el poder político. Regularmente la historia ha caracterizado esta relación en términos de conflicto. No obstante, esta vía de rendición de cuentas ha ido transformando una democracia participativa con sentido electoral, hacia una democracia más deliberativa con ciudadanos interesados en mejorar la calidad de la gestión de los asuntos públicos a cargo del gobierno. De esta forma, una democracia que delibera, se comunica con sus ciudadanos y sus relaciones entre gobierno y ciudadanía oscilan entre el conflicto, el control y la colaboración para la incidencia y la influencia desde lo social, en el espacio público. La rendición de cuentas societal busca monitorear el comportamiento de los funcionarios públicos $y$, si es necesario, exponer y hacer visibles actos ilegales.

La rendición de cuentas horizontal alude a los controles entre los poderes y equilibrios del ejercicio del poder, donde las decisiones son fragmentadas mediante un conjunto de competencias para evitar depositar en un solo individuo todas las decisiones. El poder absoluto se encuentra libre de controles y se caracteriza por la nula participación de ciudadanos y la existencia de gobernados que actúan como súbditos. Dividir el poder político está en el centro de una democracia, y la rendición de cuentas es un mandato constitucional. Aun las poliarquías latinoamericanas, como denomina Robert Dahl a las democracias, exhiben un alto déficit de rendición de cuentas horizontal que lleva a cuestionar la naturaleza representativa de esas democracias (Peruzzoti y Smulovitz 2002).

En suma, la rendición de cuentas vertical electoral alude a instrumentos vinculados al acceso al poder, mientras que la rendición de cuentas societal y horizontal, aluden a mecanismos vinculados al ejercicio del poder público. Mientras la democracia representativa carezca de instrumentos de rendición de cuentas, el desarrollo social estará frenado, pudiendo afectar los avances logrados en los últimos ańos e, incluso, esto genera condiciones para una posible regresión hacia gobiernos autoritarios que concentran el poder de una manera absoluta.

Además de los principios de libertad, igualdad y control, está presente en las democracias el principio de la mayoría, especialmente cuando se refiere a la democracia electoral, donde quien gana se lleva el poder de decisión sobre los problemas y los asuntos públicos (Downs 1973). La democracia dejó de reducirse solo a procesos mediante los cuales elegir representantes políticos. Hoy se hace necesaria la apropiación ciudadana del espacio público e incidir en las arenas donde se toman las decisiones. 
Participar es disentir, pero también colaborar para establecer una nueva relación del gobierno y la ciudadanía, especialmente cuando se dispone de los avances cibernéticos y tecnológicos como los alcanzados hasta hoy.

Las nuevas formas de relacionarnos en el espacio público -físico o virtualimplican transformar la teoría democrática en práctica cotidiana, para dar vida a interacciones de comunicación e intercambio de información entre actores gubernamentales y societales, que serán clave en la formación de un modo democrático de gobernar, más colaborativo y que viene a aumentar las capacidades institucionales de los gobiernos para dar mejores resultados $\mathrm{y}$, en consecuencia, generar confianza institucional de los ciudadanos.

Con el aumento de saberes cívicos y nuevos acuerdos institucionales entre gobierno y ciudadanos, sin renunciar al control y vigilancia ciudadana, surge la gobernanza en red (Noveck 2008) como resultado del desplazamiento gradual desde un Estado unicéntrico que anteriormente concentraba la decisión pública, hacia un Estado más policéntrico, donde la decisión pública se visualiza en distintos nodos de una red, más descentralizada en los procesos, y que adopta una estructura organizacional menos jerárquica.

Facilitar la formación de un nuevo arreglo entre gobierno y ciudadanía para crear una gobernanza en red (Noveck 2009, Cruz-Rubio y Ramírez 2012), implicará deliberación y aceptar que la acción colectiva en torno a un asunto público dará mejores resultados si el discurso social está enraizado "en el ideal intuitivo de una asociación democrática en el que la justificación de los términos y condiciones de la asociación tiene lugar a través de la argumentación y el razonamiento público entre ciudadanos iguales" (Habermas 2005: 381).

La gobernanza colaborativa es más democrática cuando la arena pública mantiene condiciones de interacción entre muchos, debido a la aplicación de tecnologías basadas en el Internet y plataformas que permiten una interacción más ágil entre ciudadanos con interés desigual en los asuntos públicos. Es decir, la comunicación entre expertos y ciudadanos no expertos, que esgrimen argumentos racionales, no necesariamente los llevan a formar una comunidad política, sino solo a tejer redes, facilitar entendimientos y generar acuerdos sobre temas concretos.

Para mejorar la confianza ciudadana en las instituciones, se requiere abordar la gobernanza colaborativa desde una participación ciudadana informada, que incida en la liberación de datos públicos para ser transformados en conocimiento relevante de los temas en los cuales participa el ciudadano. Un Estado que reconoce lo anterior, fortalece los 
derechos de libertad de expresión, asociación y acceso a la información pública para construir el derecho a la verdad. La libertad de acceder a una información libre y plural se supedita a dos condiciones: una que se refiere a un contexto social en una diversidad de valores, creencias y opiniones externadas por el público ciudadano, y otra, la existencia de un marco legal que institucionalice nuevos canales de comunicación entre gobierno y ciudadanos (O’Donnell 2003).

El derecho humano a la verdad y la libertad implica que las distintas capacidades de ciudadanos interesados en participar de los asuntos públicos convergen para llevar a la práctica un esquema colaborativo, gestionar el conflicto en el espacio público y contribuir en la formación de agendas de políticas. La gobernanza colaborativa debe gestionar, necesariamente, el conflicto que se genera en las relaciones entre gobierno y ciudadanos para lograr estadios de colaboración temática. La gobernanza tiene como base de interés común, el atender un problema crítico que afecta a la sociedad en su conjunto, bajo un contexto de difícil entendimiento en el cual existe desconfianza de doble vía. De ahí se hace necesario establecer una relación en forma de red para acordar los términos bajo los cuales se resolverán los asuntos públicos en disputa (Klijn 1998).

La gobernanza hace énfasis en una relación bidireccional, donde las organizaciones de la sociedad civil son grupos formados con intereses diversos que buscan subir temas a la agenda para obtener recursos y atención del gobierno, pero también para controlar al gobierno. Por otra parte, el gobierno es un actor formado por un conjunto de interacciones, instituciones, organizaciones que siguen un conjunto de reglas jurídicas y organizacionales vigentes, para dar certidumbre a su actuación. Refiere a la suma de autoridades que disponen de recursos fiscales, humanos, políticos, administrativos, legales, entre otros, para tomar la decisión pública. La exigencia ciudadana hoy es mejorar la calidad de la decisión pública.

La gobernanza actual requiere de componentes centrales, como el consenso, la comunicación y la cooperación, para lograr la calidad deseada de los gobiernos, además de disponer de confianza y de legitimidad ciudadana para que los gobiernos sean democráticos. Cabe precisar que este arreglo institucional entre gobierno y ciudadanos no refiere a un modelo consensual, en los términos que promueve Lijphart (2000), de inclusión de las minorías electorales y partidos políticos que no fueron favorecidos por la regla de la mayoría en las elecciones. Esta forma de gobernanza sí refiere a todos los grupos sociales y coaliciones promotoras formadas desde la sociedad civil, sin fines electorales, que buscan incidir en la agenda pública. 
La falta de confianza ciudadana en las instituciones tiene raíces profundas, y cuando se revierte tal situación a través de la transparencia y la rendición de cuentas, tales cambios son, por lo general, volátiles y sujetos a vaivenes políticos. Junto con la falta de confianza, ha sido percibido que el Estado es incapaz de resolver los problemas públicos a lo largo del continente latinoamericano, justamente porque él era parte del problema, por ejemplo, la corrupción. Y ello tuvo consecuencias como el fracaso sostenido por alcanzar a satisfacer una percepción positiva de cumplimiento de las funciones públicas encomendadas por los electores y la demanda de servicios públicos de calidad y leyes que se cumplan (Evans 2007).

Para que el Estado fuera visto como solución de los problemas, se requirió de la existencia de "una mezcla de coherencia interna y de conexiones externas, a la que puede denominarse autonomía enraizada" (Evans 2007: 43), la cual se traduce en un aumento de la capacidad del Estado. Siguiendo los análisis, funcional de O'Donnell (2008) e institucional de Mann (2007) acerca del Estado, existe coincidencia en torno a cuatro elementos fundamentales que facilitarán el aumento de la confianza ciudadana: 1) eficacia de las burocracias estatales; 2) efectividad de su sistema legal; 3) el rol estatal como foco de identidad; y 4) la capacidad de filtraje por parte del Estado. Cada elemento no ha sido desarrollado a plenitud por Estado alguno y, por ello, se debe evitar atribuirlas de manera a priori. La crisis de la representación política, de ninguna manera es una crisis de la democracia o del Estado democrático, pero sí explica la ineficacia que se produce en ambas y que, además, apunta a la importancia de encontrar nuevas fórmulas que permitan ejercer el poder público en democracia.

Bajo el contexto descrito, la democracia representativa es vulnerable a la fuerza de redes de corrupción y se mantiene en permanente peligro de ser rehén de formas de representación política que le restan legitimidad al ejercicio del poder. Una mirada schumpeteriana de la democracia donde "el pueblo tan solo tiene la oportunidad de aceptar o rechazar a los hombres que han de gobernarle" (Schumpeter 1962: 362), es el extremo que debe evitarse, pues la participación ciudadana no puede devenir en una élite participativa. La representación política en una democracia como sistema de rendición de cuentas, se vincula en forma directa la pluralidad de la participación de ciudadanos en las arenas donde se toman las decisiones.

La democracia exige contar con una representación política que facilite la inclusión en las consultas para construir la decisión pública. Siguiendo el marco analítico desarrollado por Hannah Pitkin, trabajado ampliamente por Martínez (2004), se tienen cinco formas de comprender 
la representación: 1) como autorización; 2) como responsabilidad; 3) como descripción; 4) como símbolo; y 5) como actuación sustantiva.

La representación como autorización se refiere a la confianza y la seguridad que se genera a partir de un acuerdo mutuo denominado contrato social, construcción de Hobbes en el siglo XVII. Esta representación solo importa el votante como instrumento que materializa el método democrático y dotar de legitimidad al Estado, pero no es relevante el ciudadano como un sujeto de derechos en su acepción más amplia y moderna.

La representación como responsabilidad implica que todo mandato recibido en las urnas está sujeto a un proceso de rendición de cuentas. Si bien no es un concepto de carácter obligatorio con la ciudadanía, este se lleva a cabo a través de los órganos formales del Estado, en particular el Congreso o el Parlamento.

La representación como descripción refiere a las características propias del representante y los términos bajo los cuales asume el mandato conferido por votantes en las urnas electorales. Si bien es necesario para una democracia rendir cuentas y ser responsable de las decisiones tomadas en el ejercicio del cargo, no es algo que todos representantes realicen.

La representación como símbolo alude al conjunto de procesos sobre los cuales se edifican los discursos, los intereses que supone una representación basada en símbolos y, finalmente, refiere a un sistema de creencias acerca de que la representación puede estar asociada a un conjunto de rutinas y hábitos que conducen, así como las actitudes políticas que dan origen a una cultura política.

La representación como actuación sustantiva es parte de un enfoque relacional. El principio de la representación está determinado por la manera bajo la cual se vincula y se relaciona el representante con los representados. Este tipo de representación implicará rendición de cuentas, escucha activa desde el gobierno, una nueva cultura política donde el ciudadano sea independiente frente al poder y participe activamente, al menos, en la construcción de una agenda de políticas. Se trata de un esquema de comunicación creado donde existe correspondencia mutua, esto es, una colaboración de doble vía que no renuncia al control político desde la sociedad civil. Solo lo configura en otro contexto de mayor confianza.

\section{MODALIDADES DE PARTICIPACIÓN CIUDADANA}

La representación política regida por el principio sustantivo de actuación y apertura hacia los ciudadanos facilita apuntar a la existencia de diversas 
modalidades de participación ciudadana. La literatura es amplia en el espectro del conocimiento generado sobre las formas de participación ciudadana para la incidencia pública, pero aquí discutiremos solo los trabajos de Brugue et al. (2019), Delamaza (2019), Montes de Oca (2019) y Scarpeline de Castro, Nogueira Costa y Frickmann Young (2019).

Con base en los resultados de tales investigaciones, se puede inferir que la participación ciudadana se ubica en tres niveles superpuestos de interacción con el gobierno, es decir, no son mutuamente excluyentes, y según el modelo de democracia al cual se invoque, además de las variables utilizadas, hace pertinente su caracterización atendiendo lo siguiente: 1) participación ciudadana bajo democracia directa; 2) participación ciudadana bajo democracia deliberativa; 3) participación ciudadana bajo democracia normativa.

La crisis de la representación política y la falta de una expresión clara de inclusión política han generado movimientos que destacan por el llamado al uso de figuras de democracia directa, como las consultas, el plebiscito y el referéndum. Este renovado interés en las figuras de democracia directa, como anotan Brugue et al. (2019,) es consistente con lo anotado por Delamaza (2019) cuando sugiere que la existencia de estas figuras no garantiza, necesariamente, una elevada capacidad de incidir en la solución de los principales problemas de la mayoría. Esto conduce a la cuestión formulada por Delamaza (2019): ¿quiénes participan y quienes quedan excluidos de estos procesos?

Mientras que la experiencia catalana presenta cinco casos de consulta ciudadana (Bruge et al. 2019), en Chile solo ha sido posible documentar un caso de plebiscito en la comuna de Vitacura, la cual dispone de sólidas condiciones socioeconómicas. Delamaza (2019) afirma que ocurre con frecuencia que los grupos en situación de pobreza, se les dificulta participar como ciudadanos, y cuando dejan de hacerlo,

[...] sus problemas, intereses y propuestas muchas veces no alcanzan siquiera a hacerse visibles. Al contrario, los intereses dominantes en una sociedad se hacen presentes por múltiples vías ante los tomadores de decisiones, incluso sin necesidad de participar de instancias formales (Delamaza 2019: 159).

El reflejo de la desigualdad social se manifiesta en las posibilidades de incidencia y uso de las figuras de democracia directa. La baja significación y el poco valor que tiene la participación ciudadana en la propia democracia, observado a partir de los instrumentos de política pública y como un 
mecanismo que activaría ejercicios de gobierno abierto, es todavía algo que persiste.

Para que una figura democracia directa se acerque en su instrumentación a configurar una decisión en beneficio de la colectividad de forma exitosa, se requiere introducir y poner atención en los siguientes puntos: los requisitos, el proceso de información y deliberación, la organización de la votación y la gestión de los resultados (Brugue et al. 2019).

Un aspecto que se presenta en el análisis del gobierno abierto y las innovaciones democráticas que describe Montes de Oca (2019), pone de manifiesto que la diferencia entre convocar desde una posición gubernamental o desde una posición ciudadana, sí importa y pronostica, en cierto modo, un resultado que será satisfactorio en la medida que las consultas sean más ciudadanas en el doble sentido de tener a los ciudadanos como protagonistas y a los problemas de estos ciudadanos en el centro de sus objetivos, en lugar de los problemas institucionales que presentan las entidades de gobierno y que buscan resolver con ayuda de la sociedad civil (Brugue et al. 2019: 38).

La participación ciudadana bajo un esquema deliberativo, con reglas conocidas y aceptadas, sugiere un espacio institucionalizado, que procesa posiciones antagónicas o de colaboración para alcanzar a entablar un diálogo a modo de deliberación, inclusive, cuando se trata de figuras de democracia directa. Como bien anotan Brugue et al. (2019), el espíritu de una consulta, referéndum o plebiscito es que exista un amplio debate, abierto a los encuentros en asambleas de vecinos, en las plazas y espacios públicos no estatales, donde los ciudadanos vayan construyendo colectivamente su decisión.

Si solo se atiende la cita con la democracia procedimental, acudiendo a la urna para decidir un asunto público bajo lógicas de simple agregación de preferencias a determinada propuesta hecha desde el gobierno, implicará que solo se hará una consulta de trámite, donde el representante electo no pierde el control del proceso y, por tanto, no existirían las expresiones ciudadanas plenas que aborden problemas ciudadanos que conviene a toda la sociedad deliberar. Detonar la deliberación es un eje central de la participación ciudadana en la gestión de los asuntos públicos, sea mediante figuras de democracia, o mediante el uso intensivo de enfoques relacionales de administración pública, como el gobierno abierto y las innovaciones democráticas, donde la influencia y el control ciudadano son condiciones necesarias que se observan ausentes en el análisis de literatura especializada (Montes de Oca 2019). 
La participación ciudadana bajo una democracia normativa se expresa dentro de espacios institucionalizados como consejos de participación ciudadana, consejos consultivos, entre otros. La experiencia brasileña de participación ciudadana local es vasta y está sustentada en su Constitución Política desde 1988. Brasil ha destacado por marcar una ruta para adoptar esquemas participativos locales en diversos países, desde el presupuesto participativo hasta la instrumentación de figuras de democracia directa.

Scarpeline de Castro, Nogueira Costa y Frickmann Young (2019) analizaron el impacto que ha tenido la participación ciudadana en el presupuesto público de los gobiernos locales brasileños. A través del análisis de la participación ciudadana dentro los consejos ambientales municipales, los autores lograron mostrar que se aumentó la proporción del gasto municipal en la gestión de asuntos medioambientales. Este impacto positivo es evidencia que la participación ciudadana, sea en espacios institucionales o no institucionales, gubernamentales o extragubernamentales, sí hace una importante diferencia en el resultado de la gestión pública.

Por otro parte, los consejos consultivos analizados por Montes de Oca (2019) en el caso de México, son espacios institucionalizados de convergencia entre gobierno y ciudadanos sobre temas especializados, donde normativamente el gobierno busca interacciones horizontales con agentes externos ciudadanos. Estas figuras nacen como instrumentos innovadores de participación ciudadana en México, pero posteriormente han sido ampliamente cuestionadas por terminar legitimando las decisiones previamente perfiladas por el gobierno. No obstante, al tratarse de consejos cuyas decisiones no son vinculatorias o cuyas decisiones son inducidas desde el poder, cabe valorar que el cambio social ha producido ciudadanos más críticos, preparados y dispuestos a incidir en la vida pública. Por ello, es fundamental promover la deliberación y la participación en estos espacios institucionalizados y normativos de participación ciudadana en la gestión pública, para alcanzar un mayor control ciudadano del poder o bien, la colaboración con la administración pública para aumentar sus capacidades institucionales.

\section{INNOVACIÓN EN LA PARTICIPACIÓN CIUDADANA: EXPERIENCIAS ESPAÑOLAS}

Las diversas modalidades de participación ciudadana en democracia, descritas líneas atrás, conduce a cuestionar si generan confianza en las instituciones públicas. La confianza social o interpersonal implica una relación de reciprocidad construida en las interacciones sociales, mientras que la confianza en las instituciones políticas es una actitud inductiva, 
surgida de la experiencia ciudadana de vincularse con el funcionamiento de ellas (Monsiváis 2019: 3). Existe un sentido de urgencia de reconectar lo público ciudadano con lo público estatal. Resina y Güemes (2019) comparten tres estrategias para innovar desde lo social en la generación de confianza y muestran que, bajo ciertas circunstancias y condiciones, es posible abrir espacios de participación ciudadana desde dentro del gobierno.

La primera estrategia es la creación de laboratorios de gobierno abierto. $\mathrm{Y}$ en ese punto, estudian la experiencia del gobierno de Aragón, España, que abrió la puerta a la participación ciudadana creando espacios de interacción y experimentación ciudadanas, donde "se profundizan los procesos participativos, se mejora su coordinación y se fomenta y concreta el involucramiento de la ciudadanía, al potenciar espacios de confluencia y explorar nuevos modelos de acción pública” (Resina y Güemes 2019: 52).

Cuando el gobierno de Aragón adoptó el uso de herramientas tecnológicas a través de Nexo-LAAAB, plataforma de participación online, creada para mejorar la calidad de la deliberación y la representación de los procesos locales, lo hizo con el propósito de canalizar la participación ciudadana en un espacio de experimentación para el diseño de políticas públicas, similar la experiencia que ocurre en otros laboratorios de gobierno, como el GovLab en Estados Unidos o el MindLab en Dinamarca. Esta experiencia siguió pautas metodológicas para estructurar un pensamiento de diseño en cinco fases: empatía, definición, ideación, prototipado y pruebas con carácter abierto y colaborativo. Así, se incorporó un catálogo de técnicas de debate, se estableció un servicio de mediadores que operan como nodos de una red en la cual, y se instauró un proceso de registro de las actividades online de apoyo y seguimiento.

La segunda estrategia que comparten Resina y Güemes (2019) para generar confianza ciudadana es la innovación en transparencia para el combate a la corrupción. El gobierno de Valencia, España, diseñó una tecnología anticorrupción basada en herramientas digitales de carácter preventivo y pronosticar malas prácticas administrativas en la gestión pública. Complementa la estrategia seguida por el gobierno de Valencia, la participación ciudadana en la formación de los servidores públicos en temas de gobierno abierto, lo que ha sido clave para promover una nueva cultura administrativa y un nuevo perfil de servidor público. Dentro de las actividades de la segunda estrategia, destacan encuentros entre servidores públicos de nivel directivo del gobierno de Valencia en ejercicios de rendición de cuentas ante los ciudadanos. 
La tercera estrategia que se anota es la innovación en la participación ciudadana a través del programa Madrid Escucha, el cual tuvo dos ediciones, convocando, en primer término, a todos los ciudadanos y servidores públicos que desearan presentar propuestas de mejora de la ciudad y de movilidad. Una vez seleccionados los proyectos, se invitó en un segundo momento de manera abierta a todos los ciudadanos que pudieran colaborar, anotándose en mesas de trabajo para imprimir dinámicas ciudadanas a cada proyecto e impulsar la acción de especialistas y servidores públicos involucrados en la propuesta del proyecto.

Madrid Escucha, junto con Experimenta Distrito, iniciativas analizadas por Estévez y Del Campo (2019), fueron impulsados por el MedialabPrado, el cual forma parte de la empresa pública Madrid Destino, que pertenece al Ayuntamiento de Madrid. Ambas experiencias mostraron que

[...] el intercambio colectivo permite crear comunidades de aprendizaje a partir de los cuales repensar la vida en la ciudad, generando espacios de encuentro en los que la ciudadanía presenta y discute proyectos para la ciudad y sus barrios (Estévez y Del Campo 2019: 179).

Estos trabajos han mostrado que la participación ciudadana y la innovación son factores relevantes en la generación de confianza, y así disponer de mayores capacidades institucionales y ciudadanas en la solución de problemas crítico que aquejan a los ciudadanos en América Latina: la inseguridad pública, la corrupción y el desempleo.

\section{PARTICIPACIÓN CIUDADANA Y LA INNOVACIÓN PARA EL COMBATE A LA CORRUPCIÓN}

La corrupción es un fenómeno con diversas expresiones. Por ello, su combate requiere de diversas operaciones organizacionales, que tiene como responsables a distintas entidades públicas encargadas de investigarla, sancionarla, prevenirla. La comprensión de los efectos devastadores que tiene la corrupción para toda la sociedad permite sugerir que la corrupción es fenómeno multicausal, de necesaria operacionalización según el enfoque de análisis utilizado. Un enfoque está relacionado con la profesionalización y el servicio civil de carrera vigilado por ciudadanos, para evitar la captura de puestos públicos que vinculan las decisiones de autoridad en beneficio de unos cuantos y el ejercicio discrecional de los presupuestos públicos (Merino 2013: 140). Otro enfoque de compresión del fenómeno complejo, es definir la corrupción como un problema estructural que daña las reglas, procedimientos y líneas de responsabilidad, así como a las instituciones y en consecuencia, a la institucionalidad del poder (Uvalle 2018: 72), o 
bien, como conjunto de relaciones asimétricas se traducen en la reducción de los beneficios sociales, en la claudicación al mejoramiento económico y la restricción del acceso a la arena donde se toman las decisiones públicas (Valenzuela 2019: 112).

La corrupción se manifiesta en actos cometidos por individuos en el ámbito de lo público, pero también ocurre en lo privado y no son hechos aislados de un contexto social (Casar 2016: 45). En consecuencia, importa establecer mecanismos de control en organizaciones públicas y privadas. En esa línea, el trabajo de Biderbost, Boscán y Rochín (2019) avanza en la entrega de herramientas para combatir la corrupción. En específico, ellos estudian el uso de la gestión del riesgo de corrupción. Así, una serie de controles pueden incorporarse en la gestión de las organizaciones públicas y privadas. Estos controles son: 1) manuales, que requieren el concurso y participación ciudadana; automáticos, basados en aplicaciones, algoritmos y programación; 3) preventivos, centrados en la rotación de personal y el cuidado de las licitaciones; 4) detectores, enfocándose en compras justas y a precio de mercado; y 5) sancionatorios, esto es, a través de la aplicación de correctivos por conducta sancionable.

Por otra parte, la literatura especializada describe la corrupción en esos nodos a partir del indicador de su tolerancia, donde destacan dos grupos principales: 1) ciudadanos que la toleran de forma consistente brindando apoyo a políticos corruptos y evadiendo el castigo social por la estructura de valores que predomina; y 2) ciudadanos que la toleran por la existencia de condiciones para beneficiarse de la propia corrupción (Pozsgai 2015: 102).

De este modo, la participación ciudadana cumple la función de hacer visible un problema de corrupción en la esfera pública, pero también cumple la función de operar sus alternativas de solución institucionales o no institucionales. El control social será efectivo en la medida que la ciudadanía participe de manera responsable, ética y con acciones de integridad, transparencia y especialmente, rindiendo cuentas a la propia sociedad de las acciones realizadas.

La rendición de cuentas en México se ha ido gestando a través de importantes movimientos de organizaciones y comunidades formadas con interés de proponer políticas de rendición de cuentas, en el marco de un sistema federalismo mexicano que requiere ser más cooperativo, coordinado y articulado por la acción de autoridades y ciudadanos con objetivos compartidos de una agenda pública. Un sistema de rendición de cuentas emerge como un conjunto de reglas y mecanismos, que permiten 
a los ciudadanos relacionarse con los gobiernos para diseñar e implementar políticas, que cambien el rumbo de una arquitectura incompleta de instituciones y que siguen en construcción permanente, sin los cimientos de la confianza ciudadana para atravesar necesariamente las funciones de vigilancia, control, fiscalización y sanción, dentro de un fenómeno complejo de problemas interconectados (Valenzuela 2019: 113).

Tomando prestado el lente analítico de Peschard (2011: 17), los hallazgos de Valenzuela (2019) muestran la importancia de fortalecer un sistema de rendición de cuentas en el caso mexicano, a partir de cinco dimensiones. La primera es la sistémica. En efecto, las políticas de rendición de cuentas han avanzado en la creación de sistemas nacionales y viceversa, sistemas que han creado nuevas políticas de rendición de cuentas (sistemas de transparencia, archivos, fiscalización, contabilidad y combate a la corrupción). Todos los sistemas que generan políticas de rendición de cuentas, no necesariamente están interconectadas entre sí y un sistema tiene como propósito crear nuevas interacciones entre gobierno, administración pública y ciudadanos, sobre todo para mejorar la confianza ciudadana en las instituciones y en la propia democracia.

La segunda dimensión es la responsabilidad pública y la prevención. La creación del Sistema Nacional Anticorrupción mexicano tuvo origen y consecuencia en la aprobación de un conjunto de leyes que crean, definen y esclarecen las responsabilidades públicas en el combate a la corrupción. Por décadas se reservó el concepto de corrupción solo a la burocracia, poniendo en la lupa de la crítica al sector público, perdonando a empresarios y particulares que se beneficiaban de intercambios al margen de la ética, la integridad y de la propia ley. Hoy, en cambio, tanto servidores públicos como ciudadanos son sujetos de responsabilidad.

Una tercera dimensión es el fortalecimiento institucional. El Sistema Nacional Anticorrupción mexicano es el único que está encabezado por un ciudadano que no es servidor público. Se trata de un sistema que se basa en la importancia de tener un control y coordinación ciudadana, ya que la presidencia se coloca en el Comité de Participación Ciudadana. A través de los integrantes de este último, se formula la coordinación de las entidades públicas y de gobierno que forman el mecanismo denominado Comité Coordinador, creado para sentar en una misma mesa a los titulares de diversas agencias públicas y a ciudadanos.

La cuarta dimensión es la Política Nacional Anticorrupción. Ello requirió que cada entidad federativa se apropie del proceso iniciado en el ámbito nacional. El diseño de la política constituye una agenda de cuarenta 
prioridades que se ajustarán a la realidad local por los sistemas locales anticorrupción. Este proceso de apropiación requiere de la participación ciudadana, tanto institucionalizada en los comités que presiden los sistemas locales anticorrupción, como de ciudadanos organizados y no organizados que desean incidir en el combate a la corrupción. La política anticorrupción es una política de rendición de cuentas, por lo que vale destacar que la gobernanza colaborativa requiere de traducir lógicas de control, vigilancia, fiscalización del desempeño institucional de los gobiernos, en mecanismos efectivos de control que operen bajo lógicas relacionales, que ayuden a la coordinación de todos los actores y grupos involucrados en el combate y prevención de la corrupción (Mulgan 2003).

La última dimensión es la participación ciudadana en el combate a la corrupción. El entramado institucional del combate la corrupción en México se está ciudadanizando, y una apuesta hecha por el Estado mexicano a través de reservar en la legislación una presidencia ciudadana al sistema anticorrupción, tiene como meta articular y coordinar a todos los entes públicos. La expectativa es elevada, y la ruta ciudadanizada del combate a la corrupción está invadida de obstáculos y grupos interés que desean que esta opción fracase, para dejar fuera del Sistema Nacional Anticorrupción a los ciudadanos que han sido seleccionados.

Un sistema nacional anticorrupción supone un Estado democrático donde un proceso creciente de ciudadanización recupera para coordinador y gestionar los espacios públicos de decisión que fueron monopolizados por un Estado que ejercicio de un poder sin controles, en una etapa de autoritarismo en la que reservó para sí mismo, y sin rendir cuentas, la prerrogativa de decidir los asuntos públicos. Los movimientos sociales y la protesta fueron clave y necesarios, y se suman a la vía de cambio social para incidir en la eficacia y efectividad de las políticas y programas.

Hoy los ciudadanos en México deben presidir el sistema nacional anticorrupción, lo que requiere objetivos comunes de ciudadanos con el gobierno y articular una gobernanza colaborativa. La presidencia de un ciudadano en el sistema nacional anticorrupción es una forma de participación ciudadana institucionalizada, que choca frontalmente en ocasiones, con otras formas de participación no institucionalizadas, especialmente las que asumen una lógica de pertenencia social, que rechaza toda interacción con la representación institucional política como vía para la solución de los problemas públicos, priorizando la protesta y la acción colectiva (Melucci y Avritzer 2000). 
El Sistema Nacional Anticorrupción está poniendo a prueba la ciudadanización de la acción pública, lo que permitirá generar evidencia sólida acerca de cómo opera la participación ciudadana, bajo contexto de presión social, desconfianza y ejerciendo una función de coordinación de las autoridades que combaten la corrupción. El presidente del Comité de Participación Ciudadana del Sistema Nacional Anticorrupción en México y en sus entidades federativas, deberá ser autoridad en la materia. No por el cargo que ocupa sino por una ética del liderazgo que estará obligado mostrar cada presidente del Comité de Participación Ciudadana del Sistema Nacional Anticorrupción.

La participación ciudadana y la innovación exigen una democracia sustentada en la gobernanza colaborativa, que construya decisiones sobre la base de conocimiento de los asuntos públicos, exige hablar al poder con la verdad. Gran desafío para una democracia que incentiva la participación de la ciudadana.

\section{CONCLUSIÓN: SIN PARTICIPACIÓN CIUDADANA NO ES POSIBLE UN SISTEMA DE RENDICIÓN DE CUENTAS}

Los retos que se trazan en los números especiales editados por la Revista de Gestión Pública dedicados a la participación ciudadana, muestran pautas de conversación entre gobiernos y ciudadanos que aún se mantienen incipientes, porque su nivel de institucionalización es bajo y porque los ciudadanos que participan en los asuntos públicos mantienen una mirada de desconfianza hacia el gobierno y la administración pública.

Los aprendizajes generados por los autores revisados de ambos números especiales, permiten concluir lo siguiente: 1) las modalidades de participación de los ciudadanos son amplias y se pueden generar dentro y fuera del gobierno; 2) la innovación pública debe tener como propósito el incidir en asuntos críticos que merecen atención de la sociedad; y 3) la rendición de cuentas dará mejores resultados si aumentan los procesos de ciudadanización en las políticas y programas públicos.

Los desafíos son diversos y enormes. El primero es lo conceptual. La participación ciudadana es un concepto que está influenciado fuertemente por su contexto, por las herramientas que utilizan para incidir, por el mecanismo activado de la participación. Si se convoca desde el gobierno se concibe bajo parámetros institucionales. En cambio, si la invitación a participar viene desde la sociedad civil, entonces se concibe desde otro parámetro, más ciudadano y enfocado a sus problemáticas. 
Experimentar ejercicios de colaboración es una vía para superar la falta de confianza en las instituciones de gobierno, es otro gran desafío y se inscribe en el necesario intento de quienes tienen la oportunidad de participar en asuntos públicos, de construir la voz de aquellos que en razón de la desigualdad socioeconómica imperante en la sociedad, enfrentan el impedimento de materializar este derecho humano a la participación, y así, hacer llegar su voz a la arena donde se toma la decisión pública. Cuando se trata de participar en políticas de superación de la pobreza y de combate a la corrupción, el rendimiento de las políticas será más elevado, si la participación control fuertemente la discrecionalidad de la toma de decisiones.

Y aquí otro gran desafío de la participación ciudadana en el diseño e implementación de las políticas de rendición de cuentas, específicamente en una política nacional anticorrupción, es generar confianza ciudadana y así fortalecer nuestras democracias latinoamericanas. La participación ciudadana es central en la vida pública porque permite generar mayor confianza en las instituciones. Además, es importante porque la representación política tiene la oportunidad de aumentar su legitimidad, política e instrumental, al sumar a sus decisiones la inteligencia colectiva que viene de los ciudadanos, son ellos quienes conocen sus propios problemas. Sin participación ciudadana no existen gobiernos, ni democracias que puedan innovar de manera sostenida y eficiente.

Otro desafío relevante conduce a reflexionar en torno de la profundidad con la cual se generan nuevos problemas colectivos. La participación ciudadana basada en el conocimiento, y construido en clave ciudadana, podrá desarrollar estrategias de fomento a la integridad pública, una nueva cultura de la legalidad y colaborar en la formación de un nuevo perfil de servidor público, para dejar atrás fetichismos, estereotipos, mitos y falsas expectativas acerca de que todo gobierno y administración pública es corrupta e ineficiente. Existen esfuerzos importantes, aunque insuficientes todavía, de transparencia proactiva y de gobierno abierto que sugieren cierto liderazgo de servidores públicos por preocuparse de obtener un mejor desempeño ante la sociedad civil.

Otro gran desafío está en lograr que el liderazgo ciudadano se empate con un liderazgo del sector público dispuesto a entablar conversaciones a profundidad, acerca de los retos que juntos tienen por mejorar la rendición de cuentas, la prevención de la corrupción y el fortalecimiento de los espacios de interacción ciudadana. 
Finalmente, el desafío de emprender acciones entre el Estado y la ciudadanía, conjuntamente, deberá construirse sobre las bases para un sistema de rendición de cuentas, más relacional entre actores, que sustituya las jerarquías administrativas en el combate a la corrupción, por redes ciudadanas dispuestas a controlar el poder, colaborando y gestionando el conflicto emergente en una relación de coordinación y respeto mutuo entre gobierno y ciudadanos. 


\section{REFERENCIAS}

Biderbost, P., Boscán, G. y Rochin, N. (2019). La corrupción como responsabilidad compartida. Una revisión de las estrategias anticorrupción en el sector privado. Revista de Gestión Pública, VIII (2), 229-240.

Brugue, Q., Casademont, X., Gifreu, J. y Prieto-Flores, O. (2019). Consultas ciudadanas locales: Entre la legitimación gubernamental y el empoderamiento ciudadano. Revista de Gestión Pública, VIII (1), 9-42.

Campbell, D. F. (2008). The basic concept for the democracy ranking of the quality of democracy. Viena: Democracy Ranking.

Casar, A. (2016) México: Anatomía de la corrupción. Mexicanos Contra la Corrupción y la Impunidad. Ciudad de México: IMCO.

Cruz-Rubio, C. y Ramírez, A. (2012). ¿Políticas Públicas Abiertas? Apuntes exploratorios para el análisis y transformación de los diseños políticos bajo los principios del gobierno abierto. Estudio/ Working papers GIGAPP, 16.

Delamaza, G. (2019). La participación ciudadana en la superación de la pobreza: Realidad y desafíos para la democracia chilena" Revista de Gestión Pública, VIII (2), 147-178.

Downs, A. (1973). Teoría económica de la democracia. Madrid: Aguilar.

Estévez Rubín de Celis, J. I. y Del Campo, E. (2019). Madrid Escucha y Experimenta Distrito: Dos experiencias de colaboración y experimentación social. Revista de Gestión Pública, VIII (2), 179-210.

Evans, P. B. (2007). El Estado como problema y solución. En Acuña, C. H. (Ed.), Lecturas sobre el Estado y las políticas públicas: Retomando el debate del ayer para fortalecer el actual. Buenos Aires: Proyecto de Modernización del Estado, Jefatura de Gabinete de Ministros de la Nación.

Habermas, J. (2005). Factibilidad y validez. Sobre el derecho y el Estado democrático de derecho en términos de teoría del discurso. España: Trotta.

Klijn, E. (1998). Policy networks: An overview. En W. Kickert, y J. Koppenjan (Eds.), Managing complex network. Londres: SAGE. 
Lijphart, A. (2000). Modelos de democracia. Formas de gobierno y resultados en treinta seis paises. Barcelona: Ariel.

Mann, M. (2007). El poder autónomo del Estado: sus orígenes, mecanismos y resultados. En Acuña, C. H. (Ed.), Lecturas sobre el Estado y las politicas públicas: Retomando el debate del ayer para fortalecer el actual. Buenos Aires: Proyecto de Modernización del Estado, Jefatura de Gabinete de Ministros de la Nación.

Martínez, M. A. (2004). La representación política y la calidad de la democracia. Revista Mexicana de Sociología, 66 (4), 661-710.

Melucci, A., y Avritzer, L. (2000). Complexity, cultural pluralism and democracy: Collective action in the public space. Social Science Information, 39 (4), 507-527.

Merino, M. (2013). La captura de los puestos públicos. Revista Mexicana de Ciencias Políticas y Sociales, LVIII (219), 135-156.

Monsiváis, A. (2019). La calidad percibida de los servicios públicos locales y la confianza institucional en México. Región y Sociedad, 31, e1206. doi: $10.22198 /$ rys2019/31/1206

Montes de Oca, L. B. (2019). ¿Una nueva forma de gobernar? Modelo heurístico para analizar la participación ciudadana en la gestión pública. Revista de Gestión Pública, VIII (1), 71-106.

Morlino, L. (2005). Democracia y democratizaciones. Ciudad de México: Centro de Estudios de Política Comparada.

Mulgan, R. (2003). Holding power to account. accountability in modern democracies. Nueva York: Palgrave.

Noveck, B. (2008). WikiGovernment. How open-source technology can make government decision-making more expert and more democratic. Democracy: A Journal of Ideas, 7, 30-43.

. (2009). Wiki government. How technology can make government better, democracy stronger, and citizens more powerful. Washington DC: Brooking Institution Press.

O’Donnell, G. (2003). Democracia, desarrollo humano y derechos humanos. En O'Donnell, G., Iazeta, O. y Vargas Cullel, J. (Eds.), Democracia, desarrollo humano y ciudadaní. Santa Fe: Homo Sapiens. 
- (2008). Algunas reflexiones acerca de la democracia, el Estado y sus múltiples caras". Ponencia presentada en el XIII Congreso Internacional del CLAD sobre la reforma del Estado y la Administración Pública. Buenos Aires, Argentina.

Peruzzoti, E. y Smulovitz, C. (2002). Controlando la Política. Ciudadanos y medios en las nuevas democracias latinoamericanas. Buenos Aires: Temas Grupo.

Peschard, J. (2011). El papel del derecho de acceso a la información en la rendición de cuentas". En López -Ayllon, M., Merino y L. Morales (Eds.), Hacia una política de rendición de cuentas en México. Ciudad de México: Auditoria Superior de la Federación, CIDE.

Pozsgai, J. (2015). Low-level corruption tolerance: An action-based approach for Peru and Latin America. Journal of Politics in Latin America, 7 (2), 99-129.

Resina, J. y Güemes, C. (2019). Instituciones abiertas y creación de confianza: Experiencias desde España. Revista de Gestión Pública, VIII (1), 43-70.

Sartori, G. (2003). ¿Qué es la democracia? Ciudad de México: Taurus.

Scarpeline de Castro, B., Nogueira Costa L. A. y Frickmann Young, C. E. (2019). Citizen participation and local public management: The case of municipal environmental councils in Brazil. Revista de Gestión Pública, VIII (2), 211-228.

Schumpeter, J. A. (1996). Capitalismo, socialismo y democracia. Madrid: Aguilar.

Uvalle, R. (2018). Restricciones del Sistema Nacional Anticorrupción. Análisis de su diseño institucional. Ciudad de México: UNAM y La Biblioteca.

Valenzuela Mendoza, R. E. (2019). El poder ciudadano en la gobernanza del Sistema Anticorrupción de México. Revista de Gestión Pública, VIII (1), 107-140.

Recibido: 02-12-2019

Aceptación de la versión final: 30-12-2019 